\title{
3D Nanostructured Conjugated Polymers for Optical Applications
}

\author{
Raphael Dehmel, Alexandre Nicolas, Maik R. J. Scherer, and Ullrich Steiner*
}

The self assembly of block-copolymers into the gyroid morphology is replicated into 3D nanostructured conjugated polymers. Voided styrenic gyroidal networks are used as scaffolds for the electrodeposition of two poly(3,4-ethylenedioxythiophene) derivatives and poly(pyrrole). The careful choice of solvents and electrolytes allows the excellent replication of the initial self-assembled morphology into self-supporting gyroidal conjugated polymer networks. The nanostructured films are employed to fabricate electrochromic devices, exhibiting excellent color contrast upon switching, with fast switching speeds. The versatility and reliability of this method are demonstrated by the creation of switchable Fresnel zone plates, with which the focussing of light can be switched on and off.

\section{Introduction}

Conductive polymers play an important role in several key technologies, such as printed electronics, ${ }^{[1]}$ organic solar cells, ${ }^{[2]}$ batteries, ${ }^{[3]}$ and display technologies. ${ }^{[4]}$ For many potential applications the intrinsic properties of these functional materials fail however to meet demanding technological requirements. In particular, the optical response caused by a chemical trigger is often diffusion limited, causing relatively slow response times. ${ }^{[5]}$ The structure formation of these materials on small length scales greatly enhances their specific surface area, which typically improves properties that arise from surface chemistry or interdiffusion processes..$^{[6-8]}$

The formation of 3D nanostructures in soft organic materials holds several challenges, particularly with respect to mechanical stability and resistance to plasticization by small molecules. ${ }^{[9]}$ Some studies have shown that 3D architectures outperform those of lower dimensionality (e.g., nanowire arrays) in terms of their mechanical stability. ${ }^{[10,11]}$ Additionally, 3D interconnectivity guarantees the transport of charge carriers across the network.

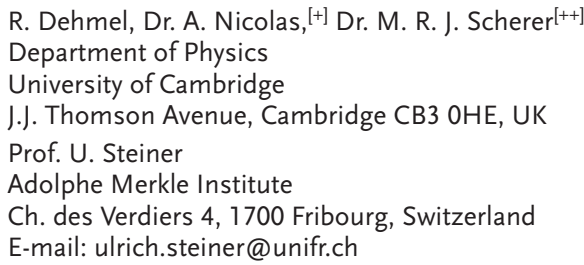

${ }^{[+]}$C.A.B. \& CONICET, Av. Bustillo 9500,8400 S.C. Bariloche, Argentina. ${ }^{[++]}$Papierfabrik Louisenthal $\mathrm{GmbH}, 83701 \mathrm{Gmund}$ am Tegernsee, Germany.

This is an open access article under the terms of the Creative Commons Attribution License, which permits use, distribution and reproduction in any medium, provided the original work is properly cited.

DOI: $10.1002 / \mathrm{adfm} .201502392$
The ideal mesoporous morphology should also exhibit interconnected pores of constant diameter for an optimized accessibility to the interfacial surface.

The double-gyroid (DG) morphology with cubic Ia $\overline{3} d$ symmetry fulfils these criteria. It is a 3D continuous equilibrium structure arising from the self-assembly of strongly segregated diblock copolymers, ${ }^{[12,13]}$ which comprises two interwoven single-gyroid networks with $I 4_{1} 32$ symmetry. These networks are related by inversion and are separated by a so-called matrix phase. A significant advantage of the DG structure is the self-supporting nature of all constituent phases, which facilitates the synthesis of free-standing networks and thus greatly increases the number of possible fabrication pathways. Moreover, the pores as well as the surrounding struts are uniformly sized and well-ordered, ensuring interconnectivity of both networks across macroscopic dimensions.

The replication of 3D nanonetwork morphologies into functional materials separates the optimization of a self-assembled template (such as a DG morphology) from the synthesis of the functional material that fills the template. While well established for metals ${ }^{[14]}$ and metal-oxides, ${ }^{[15]}$ it is also promising for the nanostructured synthesis of intractable organic materials. The replication of self-assembled templates is particularly interesting for conjugated polymers, the synthesis of which does not allow a precise control over their 3D morphology. Compared to inorganic templates, which require corrosive media for their chemical degradation, ${ }^{[16]}$ soft organic templates offer the possibility of a mild template removal by dissolution. Recently, Cho et al. have demonstrated the microemulsion synthesis of conducting poly(3,4-ethylenedioxythiophene) gels with a DG morphology. ${ }^{[17]}$ Retrieving a mesoporous aerogel from this precursor for application in functional devices is however virtually impossible because the gel itself is unstable and undergoes a transition from the gyroidal to the lamellar phase at $30^{\circ} \mathrm{C}$. In contrast, replicating mesoporous, DG-structured polymer templates into conjugated polymers may afford denser and therefore more stable functional materials.

Compared to the replication of inorganic materials, template-assisted polymer synthesis presents many challenges, as it requires a complex multistep wet-chemical process involving several orthogonal solvents that are highly specific to either the template or the synthesized replica. Furthermore, synthesis reactions have to be chosen so that the template remains inert. The following requirements are essential:

First, to form the mesoporous polymeric template, one of the copolymer blocks must be removed without affecting the 
remaining phase. Second, the functional polymer material must be electrosynthesized from solution without altering the template. Third, the latter must be dissolved in a way that does not jeopardise the delicate structure of the functional material. Finally, the mesoporous conjugated polymer electrode must be infiltrated with an electrolyte that does not affect it, structurally or chemically.

A major challenge is the identification of a suitable solvent for each processing step. The significantly increased surface-area-to-volume ratio of the $3 \mathrm{D}$ nanopatterned material, which is the primary desired feature, also has detrimental effects during sample synthesis. In particular, upon exposure to even a poor solvent, a certain surface layer of the polymer will be altered (e.g., swollen). Compared to the bulk, this affects a much larger volume fraction of a nanostructured material. For solvent-induced surface plasticization that is comparable to the dimension of structural network elements, structure loss will ensue even for solvents that do not dissolve the bulk polymer. ${ }^{[9]}$ Furthermore, even limited surface swelling will reduce the porosity of the nanostructured architecture, preventing its infiltration with additional functional components.

Here, we present a versatile synthesis route to $3 \mathrm{D}$ nanostructured conjugated polymer films on transparent electrodes, using polymeric templates made from poly(styrene) or poly(4-fluorostyrene). Three different conjugated polymers were synthesized: poly(3,4-ethylenedioxythiophene) (PEDOT), poly(3,4-ethylenedioxythiophene methanol) (PEDOT-MeOH), and poly(pyrrole) (PPy). To investigate the impact of the 3D nanopatterning on the material properties, the electrochromic performance of DGstructured PEDOT-MeOH was compared to nontemplated films. Finally, the compatibility of the presented synthesis pathway with other microfabrication techniques, as well as the micron-scale accuracy of the electrodeposition step, were demonstrated by the fabrication of electronically switchable Fresnel zone plates.

\section{Results and Discussion}

\subsection{Synthesis of Gyroid-Structured Conjugated Polymer Devices}

The synthesis strategy and the used polymers are shown in Figure 1. The route toward nanostructured conjugated polymer films consists of four stages (see the Experimental Section):

1. Manufacturing of the DG self-assembled polymer morphology: Poly(styrene)-block-poly(D,L-lactide) (PS-b-PLA) block copolymers were spin-cast onto fluorine-doped tin oxide (FTO) substrates and briefly annealed to form a DG structure.

2. Removal of one of the polymer phases to yield a mesoporous scaffold: The PLA phase was selectively degraded in an aqueous sodium hydroxide solution to form a nanoporous network.

3. Refilling the voided organic template with a conjugated polymer by electropolymerization: The voided DG films were refilled electrochemically with conducting polymers, using a potentiostat and a three-electrode cell equipped with a stainless steel working electrode and a saturated calomel electrode (SCE) as reference. The detail of electrochemical synthesis of PEDOT, PEDOT-MeOH, and PPy are given in the Experimental Section.
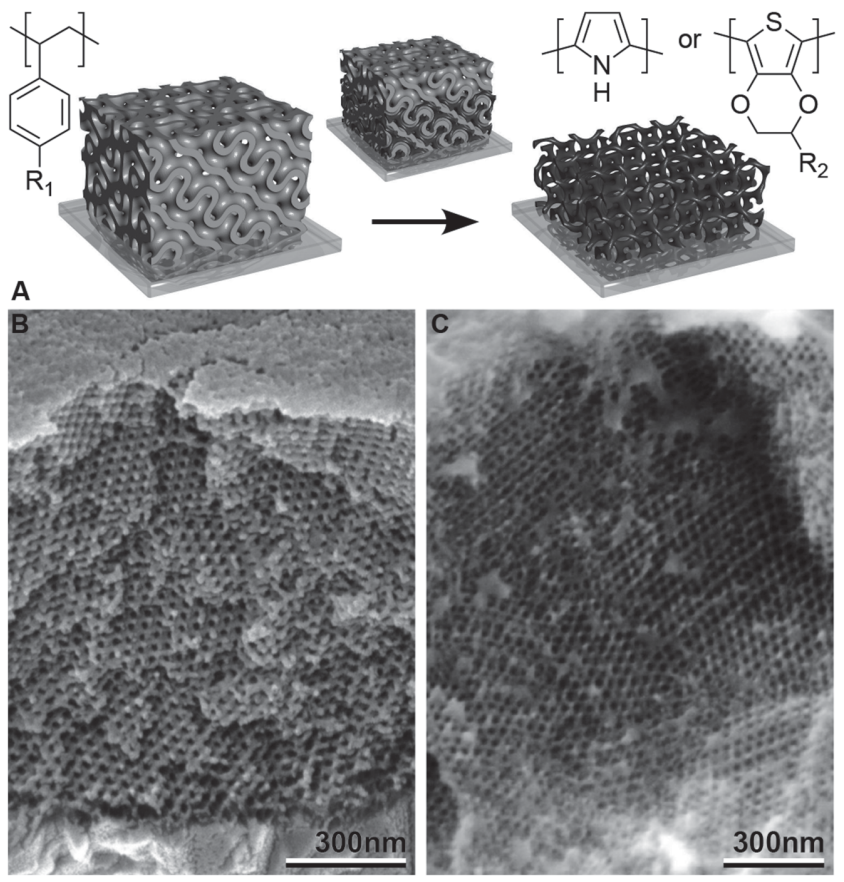

Figure 1. Preparation of DG-structured conjugated polymer electrodes. A) Schematic illustration of the gyroidal styrenic template, electropolymerization, and subsequent template dissolution yielding a freestanding conjugated polymer replica $\left(\mathrm{R}_{1}=\mathrm{H}, \mathrm{F}\right.$ and $\left.\mathrm{R}_{2}=\mathrm{H}, \mathrm{CH}_{2} \mathrm{OH}\right)$. Cross-sectional SEM images of $\mathrm{B}$ ) free-standing, mesoporous PEDOT$\mathrm{MeOH}$ and C) PPy networks.

4. Dissolution of the sacrificial scaffold polymer to yield a freestanding conjugated replica (see below).

Steps 1 and 2 have been demonstrated in numerous examples. ${ }^{[18-20]}$ The system used in this study is identical to the one examined in ref. ${ }^{[21]}$ where the structural fidelity of the assembly and etch processes were confirmed by small-angle X-ray scattering. Steps 3 and 4 are challenging and require carefully selected experimental conditions.

In step 3, protic solvents ${ }^{[1,22-25]}$ (mainly water) as well as acetonitrile ${ }^{[22]}$ have been used as electrolytes for the electrosynthesis of PPy and PEDOT derivatives. For PPy, the use of an acetonitrile-based electrolyte was reported to yield more compact films, with superior electron transfer characteristics and conductivities, presumably owing to the growing hydrophobicity of the chains during electropolymerization. ${ }^{[22]}$ Because of their denser packing, films synthesized in acetonitrile may also be more resistant to solvents than films deposited in an aqueous solution. Acetonitrile unfortunately is a solvent for polystyrene and can therefore not be used here. In contrast, protic solvents do not swell or dissolve the poly(styrene) and poly(fluorostyrene) templates. PPy was therefore electrodeposited on a FTO-coated glass submersed in water, which yielded high-quality, pitch black films.

The synthesis of PEDOT and PEDOT-MeOH in water was not successful, yielding nonuniform films. Instead a 1:1 mixture of water and methanol was used as electrolyte, which produced vividly blue films. All films exhibited a DG morphology with a strut radius of roughly $5.5 \mathrm{~nm}$. They were obtained in a 
highly controllable and reproducible way. Note that in an alternative attempt PEDOT derivatives were electrodeposited in trifluoroethanol, with unsatisfactory results.

Step 4, the dissolution of the styrenic template without affecting the synthesized PPY or PEDOT proved more challenging. Toluene is a poor solvent for PEDOT and Jones et al. have demonstrated the synthesis of nanoporous PEDOT with a strut radius of around $50 \mathrm{~nm}$ using a toluene-containing solution. ${ }^{[26]}$ Scanning electron microscopy (SEM) analysis of the DG-structured PEDOT deposits that were immersed in toluene however revealed significant structural degradation (compare Figure S4 to Figures S5 and S6, Supporting Information). The loss in pore size uniformity is ascribed to a plasticization of a nanometer-thick surface layer ${ }^{[9]}$ by the solvent. In nanometer-sized struts, this results in the decay of the entire periodic DG structure into a nonordered mesoporous morphology (Figure S4, Supporting Information). The fact that the structure does not completely collapse suggests that the penetration depth of toluene in PEDOT is comparable to, but somewhat smaller than, the DG strut radius.

Hansen solubility parameters (see Supporting Information, Tables T1 and T2) were instead employed to identify suitable solvents for polystyrene that affect PPY and PEDOT only very little. The screening of possible candidates led to the choice of xylene and a mixture of diethyl ether and hexane (2:1 mixing ratio by weight), respectively. The use of these solvents resulted in free-standing PPy and PEDOT gyroids (Figure 1B,C). The formation of uniform porosity in the samples was confirmed by electron microscopy (Figure 1 and the Supporting Information).

\subsection{Optical Properties}

\subsubsection{Coloration Contrast}

The effectiveness of nanostructure formation was tested by measuring the electrochromic response of replicated PEDOT DGs.

A series of PEDOT-MeOH films with varying film thicknesses (controlled by the deposition charge density) were produced and their optical transmittance $T$ at a wavelength of $532 \mathrm{~nm}$ was measured in a $0.2 \mathrm{~m}$ solution of lithium perchlorate in deionized water during an applied voltage sweep between $-2.1 \mathrm{~V}\left(T_{\mathrm{c}}\right)$ and $+1.0 \mathrm{~V}\left(T_{\mathrm{b}}\right)$. The variation of $T$ on the deposition charge density is shown in Figure 2A and Figure S9 (Supporting Information). The device synthesized with $630 \mathrm{mC} \mathrm{mm}^{-2}$ exhibited an optimal coloration contrast $\Delta T=T_{\mathrm{b}}-T_{\mathrm{c}} \approx 48.5 \%$ and was therefore selected for further characterization (Figure $2 \mathrm{~B}-\mathrm{F}$ ). For comparison, a PEDOT-MeOH film was prepared under similar conditions but in absence of a DG template (Figure S10,
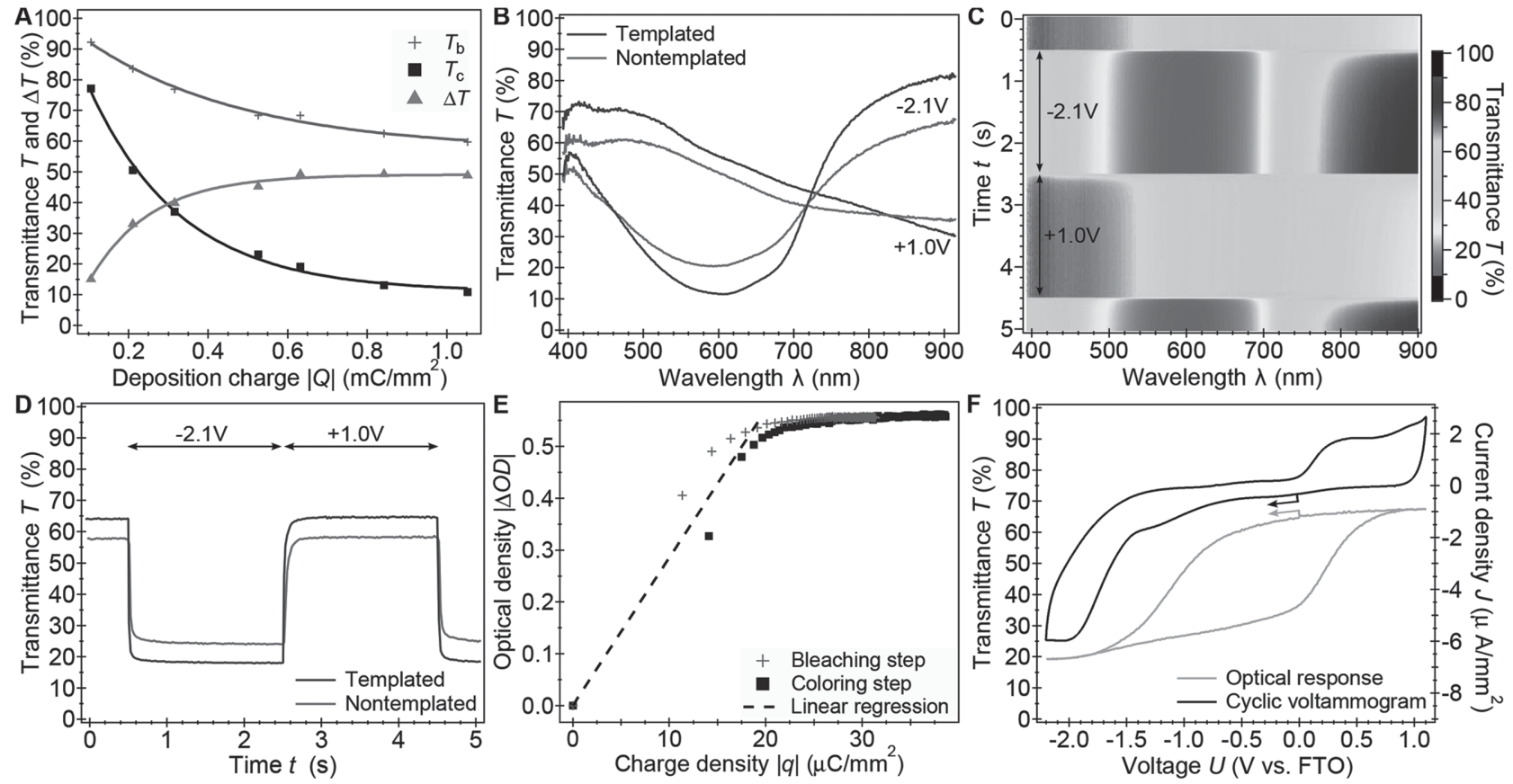

Figure 2. Electrochromic performance of PEDOT-MeOH films in an aqueous electrolyte containing $0.2 \mathrm{~m}$ lithium perchlorate. A) Variation of the extremal transmission states $\left(T_{c}\right.$ at $-2.1 \mathrm{~V}$ and $T_{\mathrm{b}}$ at $\left.+1.0 \mathrm{~V}\right)$ at $\lambda=532 \mathrm{~nm}$ with overall film deposition charge (Figure S9, Supporting Information). The film electrosynthesized with $630 \mathrm{mC} \mathrm{mm}$, which corresponds to a film thickness of about $520 \mathrm{~nm}$, showed an optimal coloration contrast of $\Delta T=T_{\mathrm{b}}-T_{\mathrm{c}}=48.5 \%$. B) Optical transmittance spectra of the colored $(-2.1 \mathrm{~V})$ and bleached $(+1.0 \mathrm{~V})$ state. A nontemplated film prepared with the same deposition charge showed a lower coloration contrast of only $\Delta T=34.2 \%$ at $\lambda=532 \mathrm{~nm}$. C) Transmittance variation upon alternating $2 \mathrm{~s}$ potential steps $(-2.1 \mathrm{~V}$ and $+1.0 \mathrm{~V})$ recorded with a $20 \mathrm{~ms}$ integration time, visualizing a consistently fast switching response over the entire visible spectrum. D) Time-resolved switching behavior of DG-structured and nontemplated PEDOT-MeOH films at $\lambda=532 \mathrm{~nm}$. The DG device exhibited characteristic switching times $\tau$ of 23 and $14 \mathrm{~ms}$ for the coloring and bleaching process, respectively. The corresponding values for the nontemplated sample were 40 and $29 \mathrm{~ms}$. E) Optical density $|\Delta \mathrm{OD}|$ at $\lambda=532 \mathrm{~nm}$ versus charge density $|q|$ calculated from (D) and the switching current (Figure S12, Supporting Information). A linear regression of the combined bleaching and coloration cycles gives an average CCE of $285 \mathrm{~cm}^{2} \mathrm{C}^{-1}$ for a $94 \%$ completed switch. F) Optical transmittance response at $\lambda=532 \mathrm{~nm}$ as function of the applied cell potential and corresponding CV recorded for the 24th switching cycle at a $0.1 \mathrm{~V} \mathrm{~s}^{-1}$ scan rate. 

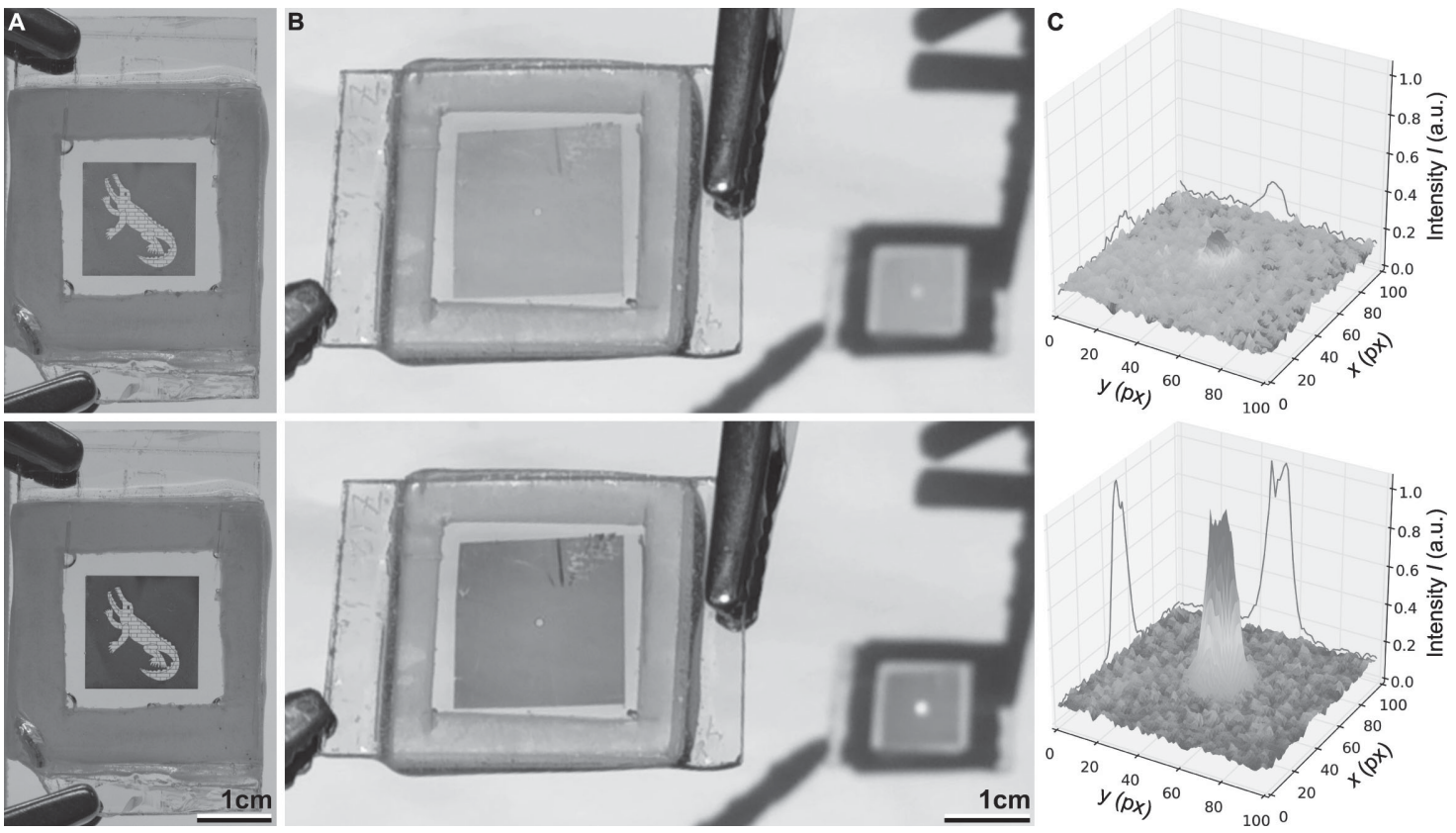

Figure 3. DG-structured PEDOT-MeOH electrochromic devices. A) Large-area device in the bleached (top, $+1.0 \mathrm{~V}$ ) and colored state (bottom, $-2.1 \mathrm{~V}$ ) showing the logo of the Cavendish Laboratory. Both states exhibit homogeneous coloration throughout the entire electrochromic area. B) An electronically switchable zone plate device exposed to sunlight (Figure S14, Supporting Information). Since the focal point is determined by the geometry of the Fresnel ring pattern, sun light is partially focused onto a focal spot at a distance of $25 \mathrm{~cm}$. In the colored state (bottom) the focal intensity of green light $(\lambda=532 \mathrm{~nm})$ was much more pronounced compared to the bleached state. Note that devices (A) and (B) were photographed with different cameras under different lighting conditions. This and the fact that only $50 \%$ of the sample in (B) contained electrochromic material led to the difference in color contrast in the two images. C) 2D intensity profile of the focal plane of a PEDOT-MeOH zone plate illuminated with a collimated beam of green light $(\lambda=532 \mathrm{~nm}$ ). The profile was recorded with Canon EOS 450D sensor (exposure $=1 / 4000 \mathrm{~s}$, ISO-400, 1 px $=5.2 \mu \mathrm{m}$ ). See Figure S15 (Supporting Information) for the experimental setup. In the colored/focusing state (bottom) the intensity of the focal spot was increased by more than a factor of 3 compared to the bleached state (top). The focal spot was very well defined, with a full width at half maximum of $\approx 72 \mu \mathrm{m}$.

Supporting Information). The deposition rates were $0.82 \mu \mathrm{m}$ and $0.31 \mu \mathrm{m}$ per $\mathrm{mC} \mathrm{mm}{ }^{-2}$ for the templated and nontemplated films, respectively. Note that for an identical charge per area, the nontemplated film should be thinner due to the lack of porosity.

Figure 2B shows the transmission spectra of both samples in the colored/reduced ( $-2.1 \mathrm{~V}$ vs FTO) as well as the bleached/oxidized state $(+1.0 \mathrm{~V}$ vs FTO). Here, the nomenclature of the two states relates to the visible part of the spectrum; for wavelengths above $\approx 720 \mathrm{~nm}$ the switching behavior is inverted. In the reduced state the films have a dark blue/purple appearance that changes to pale blue on oxidation (Figure 3A). As expected, the spectra of templated and nontemplated films are similar. The mesoporous sample exhibits a higher coloration contrast across the visible spectrum: the 3D nanostructured electrochromic electrode benefits from an enhanced coloration contrast of $\Delta T \approx 48 \%$ compared to 34\% for the nontemplated film (also see Figure 2D).

An important figure of merit for electrochromic devices is the composite coloration efficiency $\mathrm{CCE}=\Delta \mathrm{OD} / q$, corresponding to the electrical charge $q$ required to drive a change in the optical density $\Delta \mathrm{OD}=\log \left(T / T_{0}\right){ }^{[27]}$ The nanostructured devices had a high CCE value of about $285 \mathrm{~cm}^{2} \mathrm{C}^{-1}$ to reach $94 \%$ of the total change in transmittance at $\lambda=532 \mathrm{~nm}$ for applied switching potentials of -2.1 and $+1.0 \mathrm{~V}$ (Figure 2E). This CCE value lies in the upper part of the previously published range of 180 and $360 \mathrm{~cm}^{2} \mathrm{C}^{-1[28-30]}$ and is about twice as high as CCE $=140 \mathrm{~cm}^{2} \mathrm{C}^{-1}$ measured for the nontemplated film (Figure S10E, Supporting Information).
For most electrochromic applications a precise control over the transmittance level is also important. The DG structured device shows a sensitive transmission response to a varying cell potential swept between -2.2 and $+1.1 \mathrm{~V}$ (Figure $2 \mathrm{~F}$ ). The transmittance change correlates well with the onset of electrical current passing through the cell. The main transmittance change occurs in the -1.5 to $+0.5 \mathrm{~V}$ voltage range at fairly low currents, with only a small transmittance change at significantly higher currents outside this range. These high currents do not arise from doping or dedoping the conjugate polymer, but are related to unwanted side reactions, such as electrolyte degradation or water splitting. The inferior electrochemical stability of water compared to other commonly used electrolytes, such as acetonitrile or propylene carbonate, limits achievable CCE values. DG-structured devices might therefore exhibit significantly enhanced CCE values with more stable electrolytes.

The results in Figure 2 were reproducible, subject to only minor fluctuations. These probably originate from variations in environmental conditions such as ambient temperature and humidity during the sensitive infiltration and electropolymerization processes.

\subsubsection{Switching Rates}

Figure 2C and Figure S10C (Supporting Information) show the time-resolved response of both templated and nontemplated films, respectively, under alternating potentials of +1.0 and 
$-2.1 \mathrm{~V}$. The DG displays sharp and distinct transitions between the colored and bleached state. The switching behavior is analyzed in more detail at $\lambda=532 \mathrm{~nm}$ (Figure 2D). The DG device shows characteristic response times of $\tau_{\mathrm{b}}=14 \mathrm{~ms}$ during the oxidation step and $\tau_{\mathrm{c}}=23 \mathrm{~ms}$ for the reverse process, as determined by fitting the function $T(t)=T_{0} \pm \Delta T \cdot e^{-t / \tau}$ to the time-dependent transmittance data, where $T_{0}$ is the transmittance value of the colored state. In both switching directions the nanostructured device is roughly twice as fast as the flat film with characteristic times of $\tau_{\mathrm{b}}=29 \mathrm{~ms}$ and $\tau_{\mathrm{c}}=40 \mathrm{~ms}$. Despite their large thickness, the faster response of the templated films is not surprising. Since the few-nanometer-thin gyroid channels are electrolyte filled, the doping and dedoping processes mainly involve ionic diffusion over only nanometric distances. Increasing the thickness of the nontemplated film beyond $\approx 200 \mathrm{~nm}$ should increase the response time and reduce the CCE. In contrast, time constants should be much less affected by the thickness of nanostructured films and CCEs should increase with increasing film thickness.

High switching rates have been reported earlier, with values below $10 \mathrm{~ms}$ for a PEDOT nanotube array. ${ }^{[16]}$ Again, this most likely arises from the use of an acetonitrile-based electrolyte. ${ }^{[31]}$ This indicates that the choice of electrolyte significantly affects the quality of the device, and that there is considerable advantage to using lithium perchlorate in acetonitrile rather than in water.

In principle, this chemical advantage could be combined with the structural benefits of the templated films reported here. Unfortunately, PEDOT and PEDOT-MeOH films synthesized in this study are not sufficiently stable in an acetonitrilebased electrolyte. A rapid decay of the electrochromic performance between consecutive switching cycles was observed when using acetonitrile and subsequent SEM analysis revealed the collapse of the initially free-standing DG structure. In contrast, the DG device was stable over dozens of switching cycles when operated in aqueous electrolytes (Figure S11, Supporting Information). Only after several hundred cycles the electrochromic performance degraded, concomitantly with the final collapse of the structure, as confirmed by SEM analysis.

The reduced chemical stability of the PEDOT DGs in acetonitrile electrolytes, compared to the study of Cho et al., most likely results from their manufacture by electrodeposition in a waterbased solvent, as explained above. Indeed, an attempt of electrodeposition and electrochemical cycling using ionic liquids (e.g., triethylsulfonium bis(trifluoromethylsulfonyl) imide) yielded films with enhanced chemical resistance, but at the expense of considerably slower switching rates and reduced optical contrast. This is in line with the optical performance of our DG-structured devices using an aqueous electrolyte, significantly exceeding those of ref. ${ }^{[16]}$ where reflectivity changes were limited to $\Delta R \approx 25 \%$.

In summary all of the examined electrolytes have distinct advantages and disadvantages in terms of switching speed, optical contrast, or chemical stability. The identification of electrodeposition and operation electrolytes that combine the advantages is therefore a highly promising route toward devices applicable in a commercial context.

\subsection{Fresnel Zone Plates}

The versatility of the DG templated manufacture route, its scalability and compatibility with other microfabrication techniques were demonstrated by the manufacturing of switchable Fresnel zone plates (Figure S14, Supporting Information). A pattern made of concentric rings has the ability to focus monochromatic light by diffraction. The level of focusing quantitatively reflects the accuracy of the ring pattern and hence the precision of the templated electrodeposition of a lithographic pattern.

Light passing through a zone plate is diffracted by alternating opaque and transparent rings. Here, the radii $r_{\mathrm{k}}=\sqrt{k \lambda f+k^{2} \lambda^{2} / 4}$ of the rings were chosen so that green light $(\lambda=532 \mathrm{~nm})$ of normal incidence shows an interference maximum at a focal length $f=250 \mathrm{~mm}$ on the optical axis. This ring pattern was manufactured by photolithography onto a FTO-covered glass slide. A voided DG film was then deposited onto this substrate and PEDOT-MeOH was electrodeposited. Since the electrochemistry took place only on the regions not insulated by the photoresist, this resulted in concentric PEDOT$\mathrm{MeOH}$ design patterns.

In the manufactured electrochemical device, a variation of the applied potential caused a change in transmittance of the opaque rings. In this way, the zone plate was switched between high and low contrast ring patterns, switching the focusing of the Fresnel lens on and off. Figure 3B shows the focusing capabilities of the electrochromic zone plate device irradiated by sunlight. In more detail, the electrochromic transmittance at $\lambda=532 \mathrm{~nm}$ changes between $\approx 18 \%$ and $\approx 65 \%$. Since the focal point of the device of the ring pattern is determined by its geometry, this cycling of the device between -2.1 and $+1.0 \mathrm{~V}$ results in a significant change in focal contrast but not in focal length, as demonstrated in Figure 3B,C.

The focusing ability was quantified directing collimated light from a filtered xenon lamp $(\lambda \approx 532 \mathrm{~nm}$, beam diameter $10 \mathrm{~mm}$ ) onto a zone plate device and the focal plane of the Fresnel pattern was recorded with the sensor of a SLR camera $(1 \mathrm{px}=5.2 \mu \mathrm{m})$ (Figure S15, Supporting Information). In this way $2 \mathrm{D}$ intensity profiles were acquired in single measurements (Figure 3C). With a full-width at half-maximum of $72 \mu \mathrm{m}$, the focal spot was well defined. The measured peak intensity ratio of about 1 : 0.3 between the colored/focusing and bleached/ transparent state was higher than estimated from the transmission values of DG-structured PEDOT-MeOH of around $0.8: 0.3$ (see the Supporting Information).

\section{Conclusion}

In summary we have presented a reliable pathway to fabricate free-standing 3D nanostructured PEDOT, PEDOT-MeOH, and PPy using styrenic templates. The fabrication route does not involve etching steps with corrosive acids and can be adapted to a variety of other conjugated polymers given that a suitable set of orthogonal solvents can be identified. The nanostructured films were employed to fabricate electrochromic devices, which exhibited excellent performance in terms of CCE and switching speed. These parameters may be further optimized by carefully adapting the electrolyte. The fabrication of the electrically switchable zone plate demonstrated the reliability and scalability of this 3D nanopatterning approach, considering in particular the large number of constraints imposed on the 
system in terms of geometry, switching contrast, and by electrolyte matrix interactions.

\section{Experimental Section}

The synthesis of micrometer thick mesoporous poly(styrene) or poly(4-fluorostyrene) films with a DG morphology has been reported elsewhere. ${ }^{[21,32]}$ Briefly, PS- $b$-PLA (molecular weight $M_{W}=21.6 \mathrm{~kg} \mathrm{~mol}^{-1}$, polydispersity index PDI $=1.09$, fraction of the PLA block $f_{\text {PLA }}=39.8 \%$ wt $)$ and poly (4-fluorostyrene)- $b$-poly (D,L-lactide) (PFS$b$-PLA, $\left.M_{n}=24 \mathrm{~kg} \mathrm{~mol}^{-1}, \mathrm{PDI}=1.10, f_{\mathrm{PLA}}=38.2 \%_{\mathrm{wt}}\right)$ were spin-cast onto FTO substrates and annealed for $20 \mathrm{~min}$ at $173^{\circ} \mathrm{C}$ to form a DG structure. The PLA microphase was selectively degraded in an aqueous sodium hydroxide solution of $\mathrm{pH} \approx 13$ to form a network with $\approx 37-38 \%$ porosity (Figure S1, Supporting Information).

The voided DG films were refilled electrochemically with conducting polymers, using a potentiostat and a three-electrode cell equipped with a stainless steel working electrode and a SCE as reference (Figure 1A). In more detail, PEDOT and PEDOT-MeOH were synthesized from a water:methanol $(1: 1)$ mixture containing $0.02 \mathrm{M}$ monomer and $0.1 \mathrm{~m}$ lithium perchlorate. Electropolymerization was conducted under potentiostatic conditions at $1.05 \mathrm{~V}$ versus SCE following a $0.5 \mathrm{~s}$ long preconditioning step at $1.55 \mathrm{~V}$ for a homogenous nucleation (Figures S2 and S3, Supporting Information). The deposited thickness was controlled by monitoring the charge passing through the cell. Subsequently, the samples were rinsed with methanol and dried at $50^{\circ} \mathrm{C}$ for $10 \mathrm{~min}$. The DG-structured conjugated polymer network was then freed by selective dissolution of the styrenic template using a diethyl ether:hexane (2:1) mixture (Figure 1B and Figures S4-S6, Supporting Information). Similarly, PPy films with a DG morphology were successfully synthesized (see Figure 1C, Figures S7 and S8, and the Supporting Information for experimental details).

PEDOT-MeOH zone plate patterns were fabricated on FTO-coated glass by modifying the substrate with a photolithography-patterned resist which locally inhibits electropolymerization. Prior to the DG template preparation a $80 \mathrm{~nm}$ thick SU-8 film was spin-cast from a 1:3 mixture of SU-8 2000.5 and SU-8 2000 at a spin speed of $6000 \mathrm{rpm}$ (ramp rate $500 \mathrm{rpm} \mathrm{s}^{-1}$ ). The samples were then soft-baked, UV-exposed for $2 \mathrm{~s}$ using a MJB4 mask aligner (SussMicroTec; $\lambda>365 \mathrm{~nm} ; 60-80 \mathrm{~mJ} \mathrm{~cm}{ }^{-2}$ ) and a chrome mask (JD Photo-Tools), post-baked, and developed for $10 \mathrm{~s}$ in SU-8 developer (propylene glycol monomethyl ether acetate). The soft- and post-bake procedures were as follows: $1 \mathrm{~min}$ at $65^{\circ} \mathrm{C}, 1 \mathrm{~min}$ at $100^{\circ} \mathrm{C}$, and $1 \mathrm{~min}$ at $65^{\circ} \mathrm{C}$.

Layered electrochromic zone plate cells were assembled via the following steps: capping with a FTO counter electrode using a precut thermoplastic gasket (Parafilm) as spacer and fusing at $50^{\circ} \mathrm{C}$ for $1 \mathrm{~min}$, infiltration with aqueous $0.2 \mathrm{~m}$ lithium perchlorate solution, and finally sealing the device with epoxy glue.

\section{Supporting Information}

Supporting Information is available from the Wiley Online Library or from the author.

\section{Acknowledgements}

R.D. and M.R.J.S. contributed equally to this work. The authors acknowledge the EPSRC EP/G060649/1 and the Swiss National Science Foundation through the NCCR Bioinspired Materials for funding. This study was supported by the Nokia Research Centre Cambridge.

Received: June 11, 2015 Revised: July 11, 2015 Published online:
[1] G. B. Blanchet, Y.-L. Loo, J. A. Rogers, F. Gao, C. R. Fincher, Appl. Phys. Lett. 2003, 82, 463.

[2] G. Li, R. Zhu, Y. Yang, Nat. Photon. 2012, 6, 153.

[3] Y. Yang, G. Yu, J. J. Cha, H. Wu, M. Vosgueritchian, Y. Yao, Z. Bao, Y. Cui, ACS Nano 2011, 5, 9187.

[4] M.-C. Choi, Y. Kim, C.-S. Ha, Prog. Polym. Sci. 2008, 33, 581.

[5] M. Deepa, A. Awadhia, S. Bhandari, Phys. Chem. Chem. Phys. 2009, 11, 5674.

[6] T.-Y. Wei, C.-H. Chen, H.-C. Chien, S.-Y. Lu, C.-C. Hu, Adv. Mater. 2010, 22, 347

[7] K. J. J. Mayrhofer, D. Strmcnik, B. B. Blizanac, V. Stamenkovic, M. Arenz, N. M. Markovic, Electrochimic. Acta 2008, 53, 3181.

[8] A. R. Shahverdi, A. Fakhimi, H. R. Shahverdi, S. Minaian, Nanomed.: Nanotechnol., Biol. Med. 2007, 3, 168.

[9] B. A. Miller-Chou, J. L. Koenig, Prog. Polym. Sci. 2003, 28, 1223.

[10] E. J. W. Crossland, S. Ludwigs, M. A. Hillmyer, U. Steiner, Soft Matter 2007, 3, 94.

[11] E. J. W. Crossland, M. Kamperman, M. Nedelcu, C. Ducati, U. Wiesner, D. M. Smilgies, G. E. S. Toombes, M. A. Hillmyer, S. Ludwigs, U. Steiner, H. J. Snaith, Nano Lett. 2009, 9, 2807.

[12] A. J. Meuler, M. A. Hillmyer, F. S. Bates, Macromolecules 2009, 42, 7221.

[13] H.-Y. Hsueh, H.-Y. Chen, M.-S. She, C.-K. Chen, R.-M. Ho, S. Gwo, H. Hasegawa, E. L. Thomas, Nano Lett. 2010, 10, 4994.

[14] M. A. Ghanem, P. N. Bartlett, P. de Groot, A. Zhukov, Electrochem. Commun. 2004, 6, 447.

[15] M. Miyake, Y.-C. Chen, P. V. Braun, P. Wiltzius, Adv. Mater. 2009, 21, 3012.

[16] S. I. Cho, W. J. Kwon, S.-J. Choi, P. Kim, S.-A. Park, J. Kim, S. J. Son, R. Xiao, S.-H. Kim, S. B. Lee, Adv. Mater. 2005, 17, 171.

[17] W. Cho, J. Wu, B. S. Shim, W.-F. Kuan, S. E. Mastroianni, W.-S. Young, C.-C. Kuo, I. I. I. Thomas, H. Epps, D. C. Martin, Phys. Chem. Chem. Phys. 2015, 17, 5115.

[18] M. R. J. Scherer, P. M. S. Cunha, U. Steiner, Adv. Mater. 2014, 26, 2403.

[19] J. G. Werner, M. R. J. Scherer, U. Steiner, U. Wiesner, Nanoscale 2014, 6, 8736

[20] S. Salvatore, A. Demetriadou, S. Vignolini, S. S. Oh, S. Wuestner, N. A. Yufa, M. Stefik, U. Wiesner, J. J. Baumberg, O. Hess, U. Steiner, Adv. Mater. 2013, 25, 2713.

[21] M. R. J. Scherer, U. Steiner, Nano Lett. 2013, 13, 3005

[22] J. M. Ko, H. W. Rhee, S.-M. Park, C. Y. Kim, J. Electrochem. Soc. 1990, 137, 905.

[23] a) C. S. C. Bose, K. Rajeshwar, J. Electroanal. Chem. 1999, 15, 2566; b) S. Aeiyach, J.-J. Aaron, M. Jouini, J. C. Lacroix, P.-C. Lacaze, Langmuir 1992, 333, 235.

[24] N. Sakmeche, S. Aeiyach, J.-J. Aaron, M. Jouini, J. C. Lacroix, P.-C. Lacaze, Langmuir 1999, 15, 2566.

[25] C. Li, H. Bai, G. Shi, Chem. Soc. Rev. 2009, 38, 2397.

[26] B. H. Jones, K.-Y. Cheng, R. J. Holmes, T. P. Lodge, Macromolecules 2011, 45, 599.

[27] C. L. Gaupp, D. M. Welsh, R. D. Rauh, J. R. Reynolds, Chem. Mater. 2002, 14, 3964.

[28] S. Bhandari, M. Deepa, A. Kumar Srivastava, C. Lal, R. Kant, Macromol. Rapid Commun. 2008, 29, 1959.

[29] G. Sonmez, H. Meng, F. Wudl, Chem. Mater. 2004, 16, 574.

[30] Z. Feng, D. Mo, Z. Wang, S. Zhen, J. Xu, B. Lu, S. Ming, K. Lin, J. Xiong, Electrochim. Acta 2015, 160, 160.

[31] M. Kateb, V. Ahmadi, M. Mohseni, Solar Energy Mater. Solar Cells 2013, 112, 57

[32] M. R. J. Scherer, L. Li, P. M. S. Cunha, O. A. Scherman, U. Steiner, Adv. Mater. 2012, 24, 1217 